\title{
A rare cause of myocardial infarction: coronary embolism in a patient with prosthetic mitral valve thrombosis
}

\author{
M. Yuce, ${ }^{7}$ F. Yavuz, ${ }^{7}$ M. Cakici, ${ }^{1}$ I. Sari ${ }^{1}$ and V. Davutoglu ${ }^{7}$
}

\section{Introduction}

The incidence of systemic embolization with mechanical valves is $1 \%$ per year [1]. Most cases present as cerebrovascular events. As the incidence of mechanical valve surgery has increased in recent years, systemic thromboembolism has become an important problem. Although coronary embolism due to mechanical valve thrombosis is encountered rarely, it is an important and serious complication.

We report here a case of embolic myocardial infarction caused by a thrombus of a mechanical mitral valve.

\section{Case report}

A 34-year-old female was admitted to the emergency department of our hospital in November 2009 with a chest pain which was compressive at the retrosternal zone and which had started 5 hours before. The chest pain of the patient was assessed to be typical of acute coronary syndrome. She had no risk factors for coronary artery disease in her medical history but it was learned that she had undergone a mitral valve replacement 2 years before because of rheumatic mitral stenosis and that no international normalized ratio (INR) analysis had been done in the last 6 months. Physical examination was normal with the presence of metallic mitral valve sound. Electrocardiography was in sinus rhythm. In the laboratory analysis, creatine kinase $\mathrm{MB}$ (CKMB) was
$50 \mathrm{U} / \mathrm{L}$ (upper limit $25 \mathrm{U} / \mathrm{L}$ ) troponin I was $4.7 \mathrm{ng} / \mathrm{mL}$ (upper limit $0.01 \mathrm{ng} /$ $\mathrm{mL}$ ) and INR was 1.6.

The patient was transferred to the coronary intensive care unit with the diagnosis of non-ST elevation myocardial infarction. Acetylsalicylic acid, beta-blockers and intravenous heparin were initiated. Coronary angiography was performed because her chest pain continued despite medical treatment. Angiography showed that the left coronary system was normal but the distal portion of the right coronary artery was totally occluded by a thrombus (Figure 1). We decided to treat the patient medically since the thrombus was in the distal portion in the vessel and intravenous tirofiban was added to the treatment.

Since the patient was young, had no coronary risk factors and had an INR of 1.6, myocardial infarction was thought to be due to coronary embolism. Transthoracic echocardiography examination revealed a functional mitral prosthetic valve. However, in transoesophageal echocardiography, there was a semi-mobile thrombus on the left atrial surface of the valve measuring $5 \times$ $5 \mathrm{~mm}$ (Figure 2).

The patient recovered after medical treatment. She was discharged with a

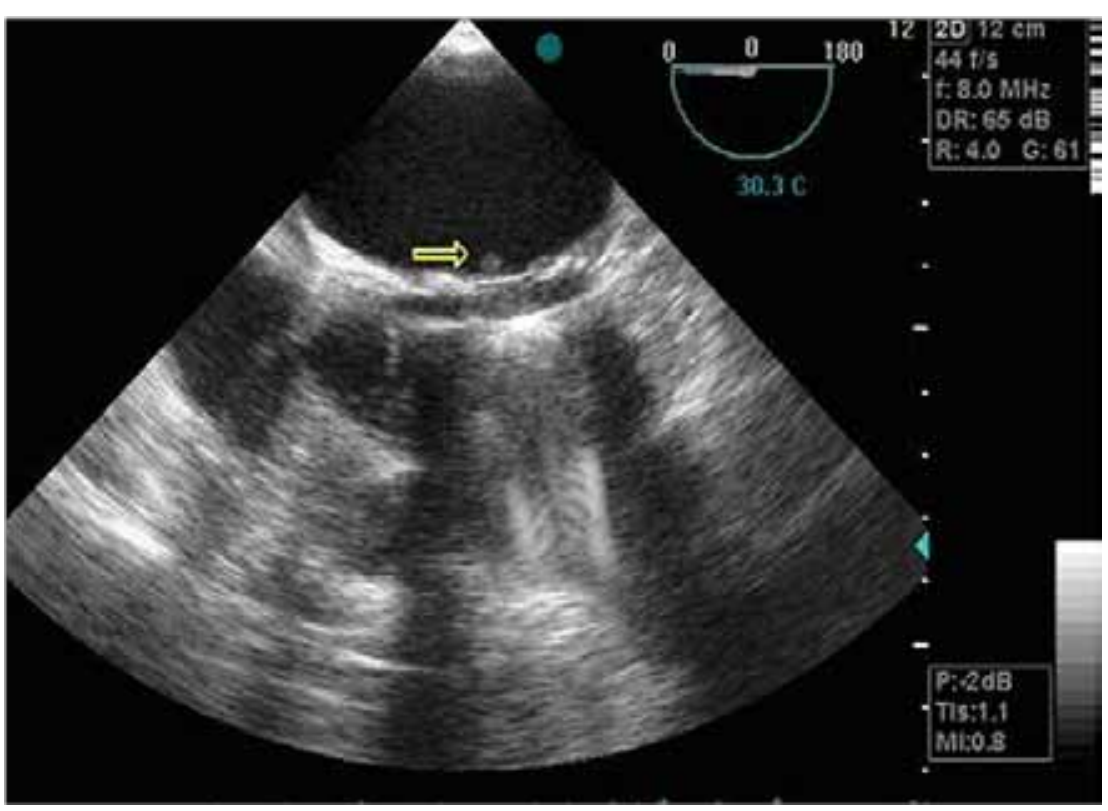

Figure 1 Thrombus on prosthetic mitral valve (arrow) 
target INR level of 2.5-3.5 and acetylsalicylic acid was added. She was free of any complications during 2 years of follow-up.

\section{Discussion}

In this paper, we report a rare case of myocardial infarction, which occurred as a result of a coronary embolism in a patient with prosthetic mitral valve thrombosis. Mechanical valve thrombosis is a serious complication [2-4]. In the pathogenesis of mechanical valve thrombosis, caged-ball valves, age $>70$ years, atrial fibrillation, depressed left ventricle systolic function, pregnancy, some drugs, carcinoma, systemic diseases, defects on the surface of the mechanical valve and insufficient anticoagulation can all play a role $[5,6]$.

Mechanical valve thrombosis causes embolism rarely. Other causes of coronary embolism include atrial fibrillation, left atrial tumours, bacterial endocarditis, atrial and ventricular mural thrombus, syphilis and pulmonary vein thrombosis [7].

Charles and colleagues reported that coronary embolism occurs in the left coronary artery in $75 \%$ of cases and three-quarters of them present with ST elevation myocardial infarction, whereas the rest present with non-ST elevation myocardial infarction [8]. In our case, the right coronary artery was involved

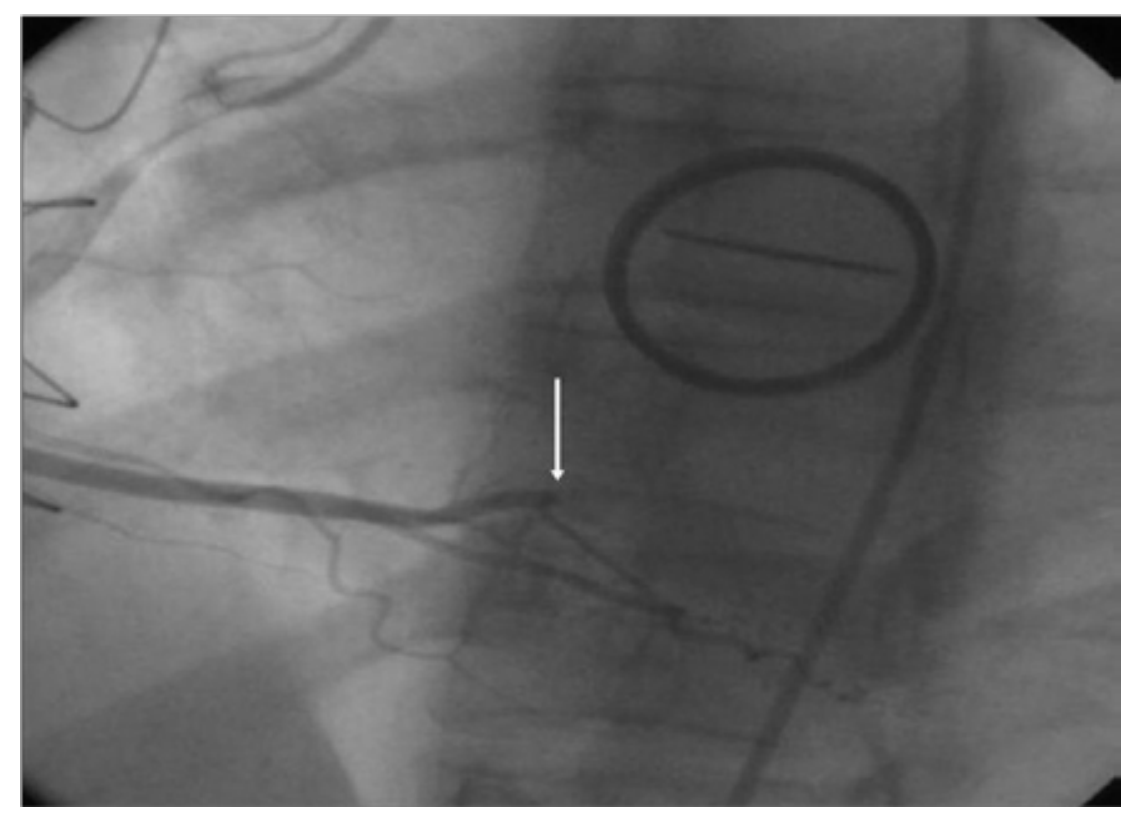

\section{Figure 2 Total occlusion of the distal right coronary artery (arrow)}

and the patient presented with non-ST elevation myocardial infarction.

There is no consensus regarding treatment of coronary embolism. Teatment options include heparin, thrombolytic agents, angioplasty and glycoprotein IIb/IIIa inhibitors [9-11]. We administered unfractionized heparin and tirofiban (glycoprotein IIb/IIIa inhibitor). We did not perform angioplasty because the obstruction was in the distal portion of the vessel and we did not administer thrombolytic agents because the thrombus on the prosthetic valve was non-obstructive and the patient was experiencing nonST elevation myocardial infarction.

Effective anticoagulation is very important for patients with mechanical valves. These patients should be educated about warfarin and possible adverse reactions with other drugs and followed regularly. In individuals presenting with acute coronary syndrome, coronary embolism should be kept in mind in those with prosthetic valves even in the absence of classical coronary risk factors, as was the case with our patient.

\section{References}

1. Cannegieter SC, Rosendaal FR, Briët E. Thromboembolic and bleeding complications in patients with mechanical heart valve prosthesis. Circulation, 1994, 89:635-641.

2. Vongpatanasin W, Hillis LD, Lange RA. Prosthetitic heart valves. New England Journal of Medicine, 1996, 335:407-416

3. Ozer $\mathrm{O}$ et al. Fibrinolytic therapy of prosthetic mitral valve thrombosis during pregnancy: three case reports and review of the literature. Clinical and Applied Thrombosis/Hemostasis, 2009, 16(4):406-413.

4. Yuce $M$ et al. Complete thrombus resolution with tirofiban in obstructive mechanical prosthetic mitral valve thrombosis. Platelets, 2010, 21(5):386-388.

5. Cannegieter SC et al. Optimal oral anticoagulant therapy in patients with mechanical heart valves. New England Journal of Medicine, 1995, 333:11-17.

6. Burchfiel $\mathrm{CM}$ et al. Left atrial dimension and risk of systemic embolism in patients with a prosthetic heart valve. Department of Veterans Affairs Cooperative Study on Valvular Heart
Disease. Journal of the American College of Cardiology, 1990, 15:32-41.

7. Charles RG, Ebstein EJ. Diagnosis of coronary embolism: a review. Journal of the Royal Society of Medicine, 1983, 76:863-869.

8. Charles RG et al. Coronary embolism in valvular heart disease. Quarterly Journal of Medicine, 1982, 51:147-161.

9. Atmaca Y, Ozdol C, Erol C. Coronary embolism in a patient with mitral valve prosthesis: successful management with tirofiban and half-dose tissue-type plasminogen activator. Chinese Medical Journal, 2007, 120:2321-2322.

10. Sial JA et al. Coronary embolism causing acute myocardial infarction in a patient with mitral valve prosthesis: successful management with angioplasty. Journal of the Pakistan Medical Association, 2009, 59:409-411.

11. Dogan $\mathrm{M}$ et al. Coronary saddle embolism causing myocardial infarction in a patient with mechanical mitral valve prosthesis: treatment with thrombolytic therapy. International Journal of Cardiology, 2009, 135:e47-48. 